\title{
Unopposed Exogenous Estrogen Administration And its Effect on The Histomorphology of Endometrium in Swiss Albino Mice
}

\author{
E.Gnanagurudasan ${ }^{1}$, S.Senthil Kumar ${ }^{2}$,Leena Dennis Joseph ${ }^{3}$ \\ ${ }^{I}$ (Ph.D Research Scholar, Department Of Anatomy, Sri Ramachandra University, Chennai, India) \\ ${ }_{2}^{2}$ (Professor, Department Of Anatomy, Sri Ramachandra University, Chennai, India) \\ ${ }^{3}$ (Professor, Department Of Pathology, Sri Ramachandra University, Chennai, India)
}

\begin{abstract}
:
Introduction: The morphology of endometrium undergoes changes in response to the sex hormones periodically. Estrogen predominantly causes mitotic activity and increases the proliferation of endometrium and progesterone antagonizes the activity of estrogen. However, factors which increase the circulating levels of estrogens such as exogenous intake of hormonal pills or endogenous increase of estrogens in response to obesity, early menarche and late menopause, chronic anovulatory cycles in PCOS can trigger proliferative lesions in the endometrium due to lack of progesterone. In the present study an attempt has been made to study the effect of unopposed estrogen administration through oral route in the histomorphology of endometrium in swiss albino mice.

Material and Methods: In the present cross sectional study 24 healthy female swiss albino mice were randomly grouped into 2 groups. Group I is the control group (6 animals) and Group II (18 animals) is the experimental group for which $17 \beta$-Estradiol had been administrated perorally for the period of 12 weeks. The animals were given normal pellet diet and water ad libitum. Result: In the present study, the endometrium showed normal morphology in the control group. Hormone treated group animals exhibited complex hyperplasia with nuclear atypia and increase in the glands stroma ratio.

Conclusion: The prolonged period of increased levels of estrogen unopposed reciprocally by progesterone causes persistent morphological changes in the endometrium leading to neoplastic changes.
\end{abstract}

Keywords: Endometrial morphology, Estrogen administration, Hyperplasia , nuclear atypia, obesity

\section{Introduction}

Endometrium of uterus undergoes cyclic changes in each menstrual cycle and its morphology is altered in response to the hormones in different phases. Estrogens stimulate the mitotic activity in the endometrium whereas progesterone antagonizes the action of estrogens. The mitosis increases gradually in the early proliferative phase of endometrium and peaks at the time of ovulation after which it declines due to the increase in progesterone levels in secretory phase [1]. The proliferation includes growth of glandular endometrium, hyperemia and increase in the contents of electrolytes, proteins and enzymes [2]. Various epidemiological studies show persuasive evidence of the link between obesity and endogenous increase in the estrogen level. Obesity increases the availability of androstendione and enhances aromatization to estrone (E1) and estradiol (E2). The production of sex hormone binding globulin reduces in obesity which increases the level of unbound estrogens. These free estrogens act on the target tissues and cause morphological changes [3]. Excess unopposed Estrogens are not only mitotic, but are carcinogenic as well which triggers neoplastic changes in the endometrium [4]. There is a continuous hyperestrogenic state in obesity which is not reciprocally opposed by progesterone which leads to hyperplastic changes in the endometrium due to the proliferative stimulus of estrogen. Persistent hyperoestrogenic state in obese women will cause proliferative changes in the endometrium and if prolonged for a long time may lead to type I or Oestrogen dependant adenocarcinoma of the endometrium. About $80 \%$ tumours are adenocarcinoma, others are non-oestrogen dependant or type II carcinoma of the endometrium. This hyperoestrogenic state can be due to other factors like nulliparity, early menarche or late menopause, chronic anovulatory cycles in polycystic ovarian syndrome (PCOS) [5, 6].

In addition to obesity, hormonal therapy with estrogens especially when unopposed adequately by progesterones causes hyperplastic changes in the endometrium. The 'unopposed estrogen hypothesis' is evident in the contraceptive pills such as sequential oral contraceptives (SOCs) which prove to increase the risk of endometrial cancer by two folds leading to their removal from the market [7,8]. Extensive research has been conducted on the use of oral contraceptives over time and proved the efficacy of combined oral contraceptives (COCs) in which there is a fixed amount of estrogens and progesterones which proved to reduce the risk of endometrial cancer by $50 \%$ than women who have not used oral contraceptives [9,10]. In premenopausal women with polycystic ovarian syndrome (PCOS), chronic anovulatory cycles lead to deprivation of progesterones to counteract the activity of estrogens [11]. Such imbalance in the estrogen- progesterone ratio for 
a long term causes histological changes in the endometrium. Thus 'the unopposed estrogen' hypothesis can ensue due to variety of reasons mentioned above. In the present study we studied the effect of unopposed estrogen administration through oral route and its effect in the histomorphology of endometrium in swiss albino mice.

\section{Material And Methods}

In the present cross sectional study 24 healthy female swiss albino mice were randomly grouped into 2 groups. Group I is the control group (6 animals) and Group II (18 animals) is the experimental group for which $17 \beta$ - Estradiol had been administrated perorally for the period of 12 weeks. The experiment was approved by the Institutional Animal Ethics Committee (IAEC No: IAEC/XLVII/SRU/476/2016) and conducted between March and July 2016 at the central animal house of Sri Ramachandra University, according to the ethical guidelines of Government of India. The animals were maintained in 12 hour light dark cycle with relative humidity of $25 \pm 2^{\circ} \mathrm{C}$ in appropriate cages containing sterile paddy husk. The animals were given normal pellet diet and water ad libitum.

\subsection{Method of administration of $17 \square$ - Estradiol:}

The experimental animals were given $17 \beta$ - Estradiol perorally by the method adopted by Jacob.O.Ström et al [12] for the period of 12 weeks. A daily dose of $1.12 \mu \mathrm{g}$ of $17 \beta$ - Estradiol was administered to the mice in the mixture of $0.312 \mu \mathrm{L}$ of sesame oil and $60 \mathrm{mg}$ Hazelnut nutella cream. The sesame oil and nutella cream act as a vehicle in which the $17 \beta$ - Estradiol is thoroughly mixed as a homogenous mixture. Prior to the start of the experiment the animals were fed nutella cream without hormone in order to train the animals to consume the cream mixture. At each day the hormone was administered to the mice individually in a separate cage to ensure uniform consumption and all the animals consumed the mixture at once. After the study period the animals were sacrificed by the inhalation of carbon monoxide and the uteri were removed and fixed in neutral buffered formalin for tissue processing. The tissues were dehydrated in ascending grades of alcohol and embedded in paraffin wax. The tissue blocks were sectioned in microtome and stained with haematoxylin and eosin for further observation.

\section{Result}

In the present study, the control group animals exhibited normal endometrium with simple columnar epithelium and endometrial glands (Table/Fig-1). Animals treated with $17 \beta$ - Estradiol showed complex hyperplasia with cytologic atypia (Table/Fig-2), nuclei are elongated and spindled out (Table/Fig-3) with crowding of glands (Table/Fig-4). There is an increase in the glands stroma ratio in the hormone treated groups (Table/fig -5). Papillary infoldings were abundant in the hormone treated animals.

\section{Discussion}

The obesity induced excess in the estrogen levels in the women with deficient progesterone to counteract leads to increase in the risk of tumor genesis in the endometrium of uterus. The aromatization of estrogen is high in obese state which favors increase in the concentration of estrogens. The excess adipose tissue in obesity becomes the substrate for the action of aromatase enzyme which triggers conversion of biologically active estrogens [13]. Thus the estrogen level is directly proportional to the body mass index. Some studies have showed reduction in the estrogen level after weight loss which suggests strong correlation with the involvement of adipose tissue in the maintenance of hormone levels in the women. Excess body weight leads to changes in the physiologic functions in the hypothalamic-pituitary-ovarian axis [4]. Endometrial hyperplasia is the proliferation of glands of irregular size and shape with an increase in the glands/stroma ratio. It is divisible further into simple and complex hyperplasia. In simple endometrial hyperplasia, there is proliferative lesion with minimal glandular complexity and crowding, more amount of stroma is seen without nuclear atypia. In complex endometrial hyperplasia, there is proliferation of glands with more complexity and crowding, less amount of stroma is present, nuclei become rounded instead of being spindled [14]. Estrogens act at molecular level and increase the transcription and growth factor signaling pathway and cause malignant changes [15]. There are several mechanisms by which obesity induce malignant transformation mainly - increased cell proliferation, cell growth from growth hormones and reduced apoptosis [16]. An overweight of more than $23 \mathrm{~kg}$ strongly increases the risk for endometrial carcinoma through hormonal excess [17]. The increase in the adipose tissue deposition leads to increase in the oxidative stress which triggers pathogenesis that develops into metabolic syndrome [16].

The experimental observation for the effects of long term exogenous estrogen administration is scarce. In the present study a novel method discovered by Jacob ström et al was adopted in which the response of the animals initially was not adequate, many animals did not start eating the nutella cream in the beginning. After due adaptation, the mice started taking the cream mixed with estrogens at once. The dosage was maintained strictly within the physiological concentration in mice as per the method of Jacob ström_[12]. The peroral 
method of administration proved to be superior than the commercial slow-release pellets and injections in terms of producing physiological concentration of estrogen in mice. Hence, in the present study in order to simulate the action of hyperestrogenic state in the otherwise healthy subject ovariectomy is not performed and peroral method was adopted.

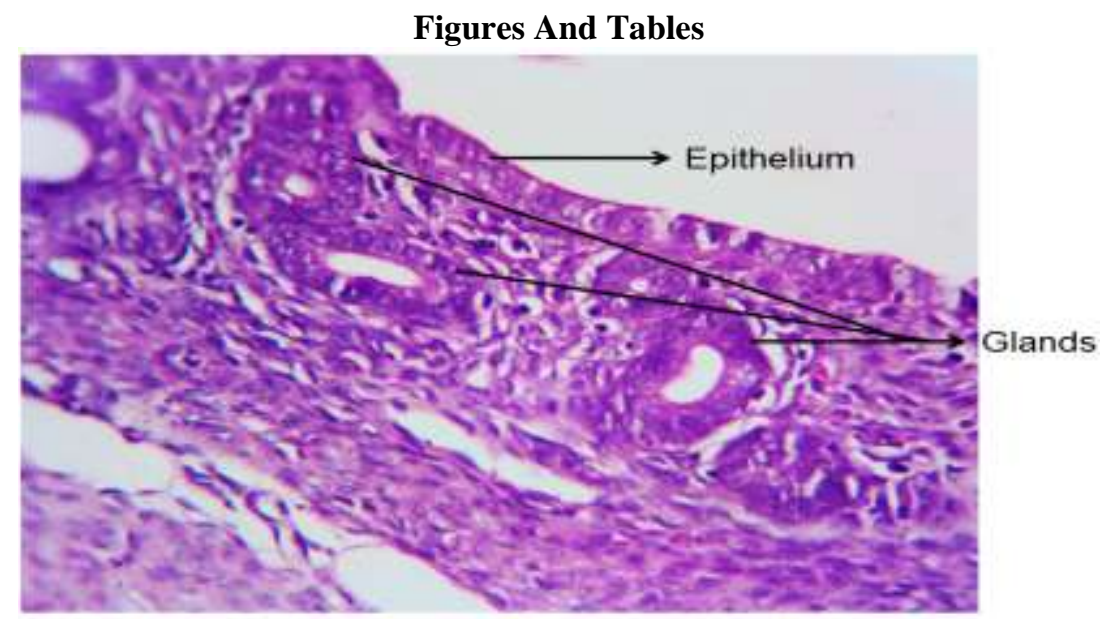

Table/Fig - 1: Endometrium showing normal appearance of epithelium and glands. (H\&E,400X)

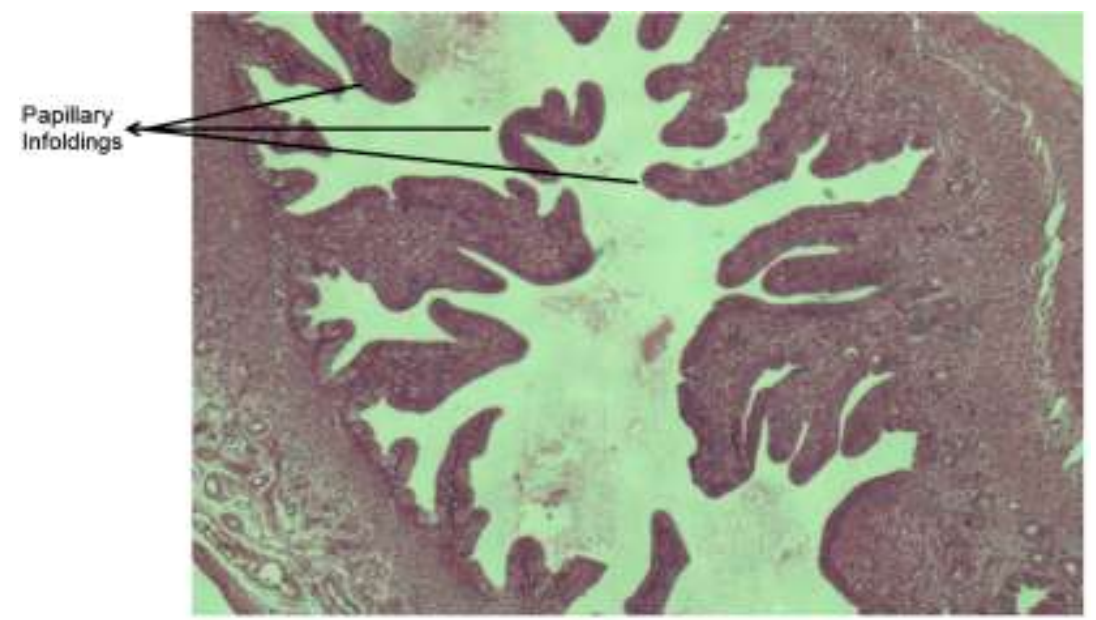

Table / Fig - 2 Hormone treated group showing complex hyperplasia with cytologic atypia. (H\&E, 100X)

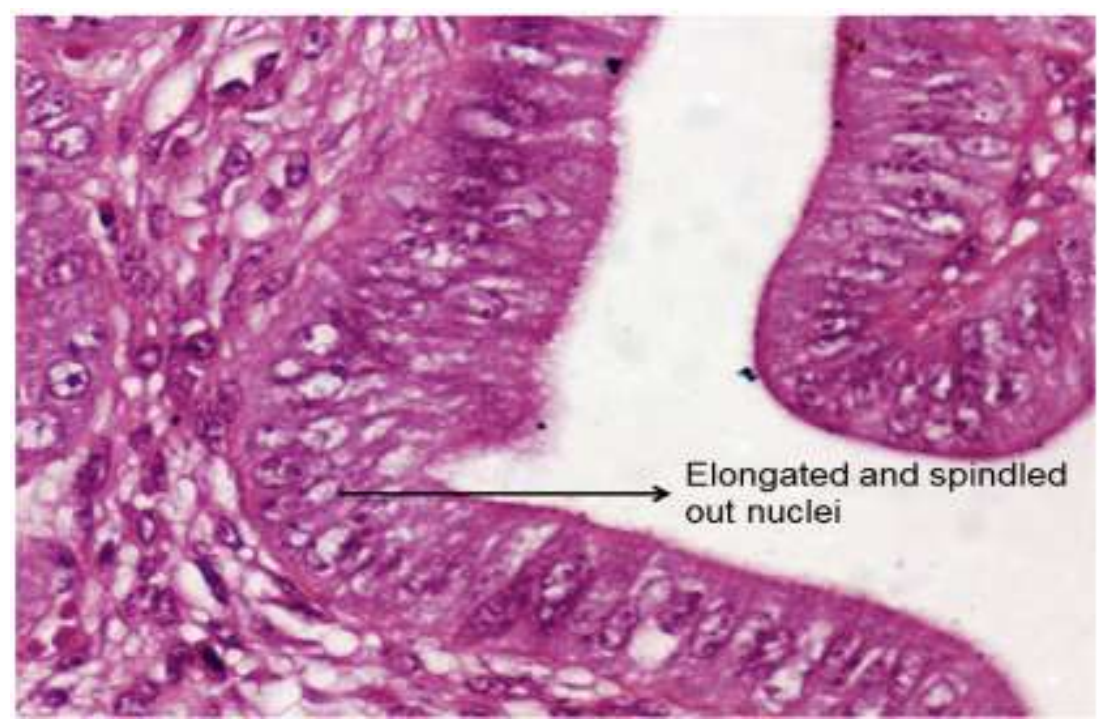

Table / Fig - 3. Elongated and spindled out nuclei in hormone treated group. (H\&E, 400X). 


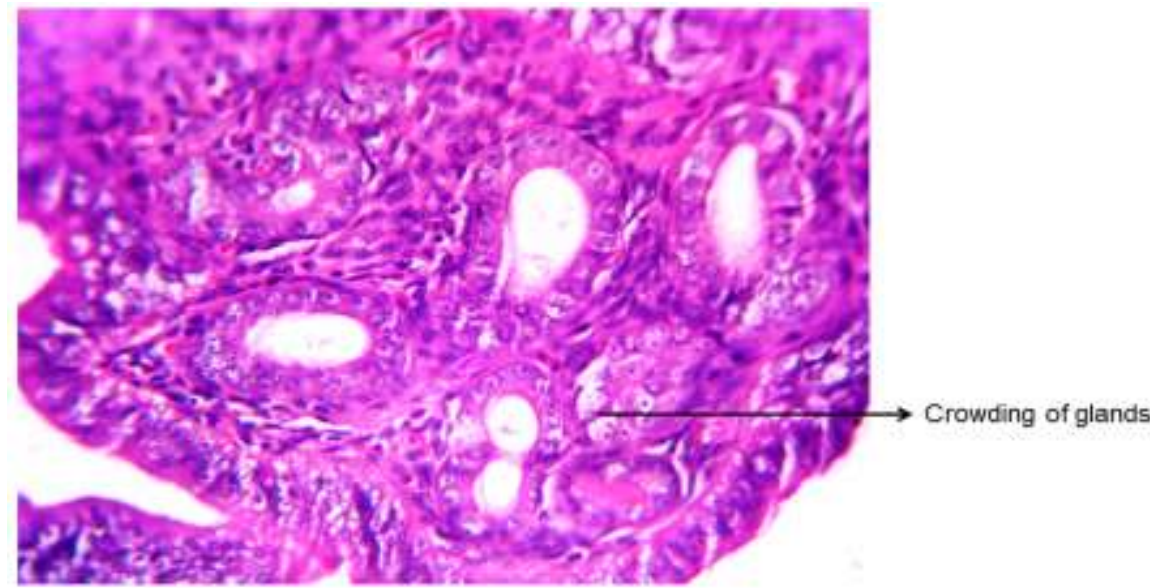

Table / Fig - 4. Crowded glands in hormone treated group. (H\&E, 400X)

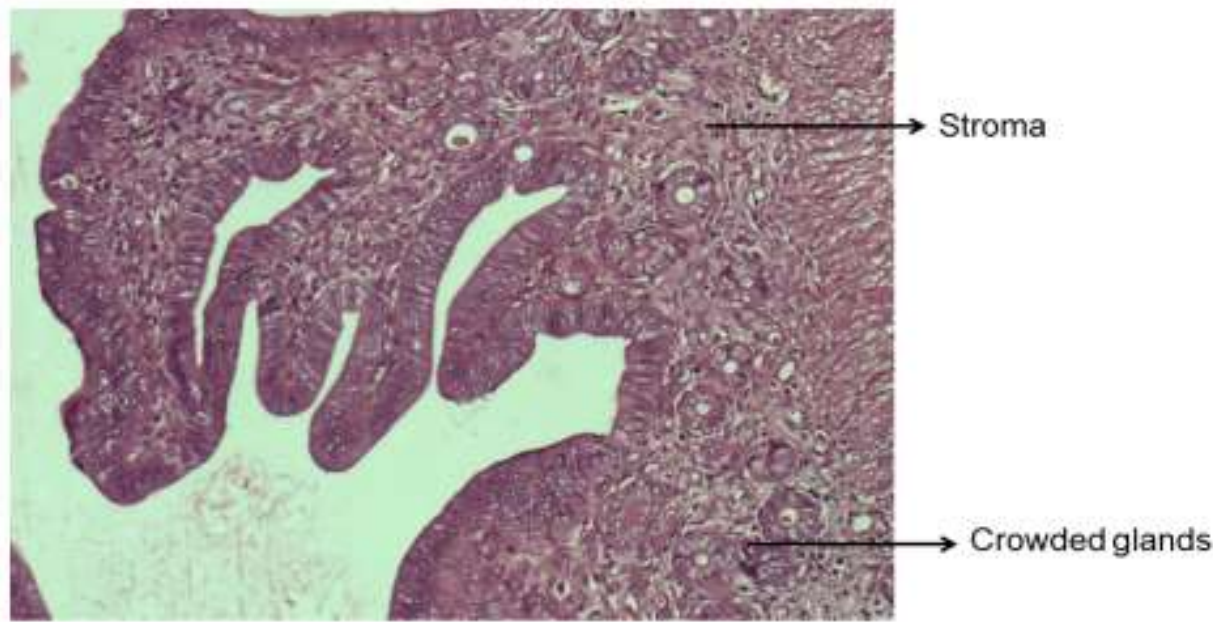

Table / Fig - 5: Hormone treated group showing increase in connective tissue stroma with crowded glands. (H\&E,100X).

\section{Conclusion}

The present study concludes that obesity is a major risk factor which increases the circulating estrogen levels and cause target tissue damage in the endometrium of uterus. The prolonged period of increased levels of estrogen causes persistent morphological changes in the endometrium leading to neoplastic changes. The simulation of such a condition in experimental animals in the present study offers good scope and throws light in the understanding of the action of estrogens in the female reproductive system.

\section{Acknowledgements}

The authors extend their thanks to Sri Ramachandra University for providing Founder-Chancellor Thiru.N.P.V. Ramasamy Udayar Research Fellowship grant for Ph.D-2014 for the present study.

\section{References}

[1]. Key JA, Pike MC. The dose-effect relationship between 'unopposed' oestrogens and endometrial mitotic rate: its central role in explaining and predicting endometrial cancer risk. Br.J.Cancer. 1998; 57: 205-212.

[2]. Guyton AC, Hall JE. Textbook of Medical Physiology, 11Ed. Philadelphia: Elsevier; 2006: 1016-1025.

[3]. Schottenfeld D. Epidemiology of endometrial neoplasia. Journal of cellular biochemistry, Supplement. 1995; 23: 151-159.

[4]. Volmer G. Endometrial cancer: Experimental models useful for studies on molecular aspects of endometrial cancer and carcinogenesis; endocrine related cancer. 2003; 10:23-42.

[5]. Morrow CP. The benefits of estrogen to the menopausal woman outweigh the risks of developing endometrial cancer. Cancer journal for clinicians. 1984; 34(4):220-231.

[6]. Montgomery BE, Daum GS, Dunton CJ. Endometrial hyperplasia: a review. Obstetrics and gynaecological survey. 2004; 59(5):368-378.

[7]. Weiss NS, Sayvetz TA. Incidence of endometrial cancer in relation to the use of oral contraceptives. New Engl. J. Med. 1980; 302: 551-554.

[8]. Henderson BE, Casagrande JT, Pike MC, Mack T, Rosario I, Duke A. The epidemiology of endometrial cancer in young women. Brit. J. Cancer. 1983; 47: 749-756. 
[9]. Kaufman DG, Shapiro S, Slone D, Rosenbert L, Miettinen OS, Stolley PD et al. Decreased risk of endometrial cancer among oral contraceptive users. New Engl. J. Med. 1980; 303: 1045-1047.

[10]. Hulka BS, Chambless LE, Kaufman DG, Fowler WC, Greenberg BG. Protection against endometrial cancer by combination product oral contraceptives. J.Amer.Med.Ass. 1982; 247: 475-477.

[11]. Kaaks R, Lukanova A, Kurzer MS. Obesity, Endogenous hormones and Endometrial Cancer Risk: A Synthetic Review. Cancer Epidemiology, Biomarkers and Prevention. 2002; 11: 1531-1543.

[12]. Ström J.O., Theodorsson A, Ingberg E, Isaksson IM, Theodorsson E. Ovariectomy and $17 \beta$ - estradiol Replacement in Rats and Mice A Visual Demonstration. J.Vis.Exp. (64), e4013, DOI: 10.3791/4013 (2012).

[13]. Crosbie JE, Zwahlen M, Kitchener CH, Egger M, Renehan GA. Body Mass Index, Hormone replacement therapy and endometrial cancer risk. A meta-analysis. Cancer Epidemiol Biomarkers Prev. 2010; 19 (2): 3119 - 3130.

[14]. Kurman RJ, Kaminski PF, Norris JH. The behavior of endometrial hyperplasia, a long-term study of "untreated" hyperplasia in 170 patients. Cancer.1985;56:403-412.

[15]. Schiff R, Massarweh SA, Shou J, Bharwani L, Mohsin SK, Osborne CK. Cross-talk between estrogen receptor and growth factor pathways as a molecular target for overcoming endocrine resistance. Clin Cancer Res. 2004; 10:331S-6S.

[16]. Jee SH, Kim JH, Lee J. Obesity, Insulin Resistance and Cancer Risk. Yonsei Medical Journal. 2005; 46(4):449-455.

[17]. Bermont L, Lamielle F, Lorchel F, Fauconnet S, Esumi H, Weisz A et al. Insulin Up-Regulates Vascular Endothelial Growth Factor and Stabilizes Its Messangers in Endometrial Adenocarcinoma Cells. The journal of clinical Endocrinology and metabolism. 2001; $86(1): 363-368$ 